\title{
Entry and Welfare in Search Markets
}

\author{
Yongmin Chen ${ }^{\dagger}$ and Tianle Zhang ${ }^{\ddagger}$
}

May 2014

\begin{abstract}
The effects of entry on consumer and total welfare are studied in a model of consumer search. Potential entrants differ in quality, with a higher-quality seller being more likely to offer consumers a high-value product. Contrary to the standard view in economics that more entry benefits consumers, we find that consumer welfare has an inverted-U relationship with entry cost, and free entry is excessive for both consumer and total welfare when entry cost is relatively low. We explain why these results may arise naturally in search markets due to the variety and quality effects of entry, and discuss their business and policy implications.
\end{abstract}

Keywords: Entry, entry cost, search, product variety, product quality JEL Classification Number: D8, L1

$\dagger$ University of Colorado at Boulder; yongmin.chen@colorado.edu

${ }^{\ddagger}$ Lingnan University; tianlezhang@ln.edu.hk

We thank Mark Armstrong, Maarten Janssen, Andrew Rhodes, David Sappington, Marius Schwartz, Guofu Tan, Mariano Tappata, Ralph Winter, participants of the $4^{\text {th }}$ Workshop on Search at the Higher School of Economics (Moscow), the 2013 Tsinghua Conference on Theoretical and Behavioral Economics (Beijing), and of seminars at Academia Sinica (Taipei), Shanghai University of Finance and Economics, University of Arkansas, University of British Columbia, University of South Carolina, and Zhejiang University for helpful discussions and comments. 


\section{INTRODUCTION}

Entry is of central importance to competition and market performance. While the efficiency of free entry under perfect competition has long been known, it is by now well recognized that, when firms possess market power, unencumbered entry has ambiguous effects on total welfare, because an entrant internalizes neither the potential consumer gain nor a rival's loss from its entry (e.g., Von Weizsack, 1980; Mankiw and Whinston, 1986; and Cabral, 2004). The standard view in economics, however, is still that more entry will boost consumer welfare. In homogeneous-product markets, industry output under Cournot competition generally expands with entry (e.g., Seade, 1980). ${ }^{1}$ Even in markets with differentiated products, where it has been argued that price-increasing entry is theoretically unexceptional, the consumer gain from greater product variety will usually dominate any potential adverse price effect (e.g., Chen and Riordan, 2008).

This paper conducts a new analysis of entry and welfare in an important class of marketsthose with consumer search, focusing especially on how entry affects consumer welfare, measured by aggregate consumer surplus. Our interest in search markets is partly motivated by the reflection that, despite the substantial progress in the economics of search, ${ }^{2}$ little attention has been paid to the effects of changes in entry conditions, and yet technological progress such as the Internet has drastically reduced entry costs in many search markets. We focus on consumer welfare because, as we shall demonstrate, the common belief that unfettered entry benefits consumers is actually misguided. This will have important ramifications for business practices as well as for antitrust and regulation policies.

We consider a market with $N$ potential entrants who differ in quality - the probability

\footnotetext{
${ }^{1}$ An important "counter-intuitive" finding is provided by Amir and Lambson (2000), who demonstrate that price can increase in the number of firms under Cournot competition. Nevertheless, as the authors point out, the assumptions needed for such an outcome, which involves an unstable equilibrium in a certain sense, are restrictive.

${ }^{2}$ Starting from the seminal work of Stigler (1961), the literature has advanced in the directions of search for the best price among competing homogeneous sellers (e.g., Stahl, 1989) and of search for the best value among competing differentiated sellers (e.g., Wolinsky, 1986).
} 
that a seller's product will match the need of each consumer (i.e., having a high value to each consumer). For example, a consumer may have a specific requirement for its product, such as a certain design feature for a car or for a house, and a high-quality seller is more likely to meet the requirement. Alternatively, a consumer may need to fix a malfunctioning product (such as a computer or a software system), or to improve a product's performance (such as the energy efficiency of a house), and a high-quality firm is more likely to find the right solution to the problem. It could also be that the consumers are input purchasers on an intermediate-good market, and a high-quality supplier is more likely to meet each buyer's quality standard for the input.

Each consumer has unit demand. A consumer's utility from purchasing a non-matched product (i.e., the low-value product) is normalized to zero. Her utility from a matched seller's product, $u$, is a (non-negative) random draw from a known distribution and is identical for all the consumer's matches. ${ }^{3}$ Each potential entrant's match probability is its private information, and the entry cost for each entrant is $k$. Potential entrants first simultaneously choose whether to enter the market by incurring the entry cost, each making the decision based on the realization of its type (i.e., its match probability), after which all sellers in the market simultaneously choose prices. The entry and price decisions are deterministic (i.e., they are pure strategies). The model is thus a dynamic game of incomplete information, and the type-contingent nature of the entry decision makes the model different from an otherwise standard two-stage entry game (e.g., Mankiw and Whinston, 1986). Notice that, ex ante, potential sellers are differentiated both vertically and horizontally. A high-quality seller, with a high match probability, is more desirable to all consumers. ${ }^{4}$ However, all matched sellers of a consumer are ex post homogeneous.

This model has a unique symmetric (perfect Bayesian) equilibrium under free entry, where

\footnotetext{
${ }^{3}$ Thus, a consumer's values for all her matched sellers are perfectly dependent. This formulation, which wil play an important role in simplifying our analysis, follows several recent papers on consumer search, including Athey and Ellison (2011), Chen and He (2011), and Eliaz and Spiegler (2011).

${ }^{4}$ If all potential sellers had the same match probability, they would differ only horizontally ex ante, in the sense that while each seller's product has a random utility, it woud have the same expected value to each consumer.
} 
any potential entrant will choose to enter the market if and only if its match probability exceeds a threshold, $t_{f}$, whereas the equilibrium market price, as in Diamond (1971), is invariant to the number of actual entrants, $n \geq 1$. At this equilibrium, a natural measure of entry is the expected number of entrants, which is a decreasing function of $t_{f}$, and $t_{f}$ in turn increases in entry cost $k$, the exogenous parameter. A reduction in $t_{f}$, or an increase in entry, expands the search options available to each consumer, but also reduces the expected quality of sellers in the market and makes it less likely for a search to produce a match. In other words, entry affects consumers through both a variety and a quality effect. It turns out that the interaction of these two effects causes consumer welfare to vary non-monotonically with $t_{f}$, and hence also with $k$. We find that consumer welfare is an inverted-U function of entry cost, first increasing and then decreasing, maximized at some critical $k^{*}$, for which the corresponding entry threshold is $t^{*}$. Moreover, when $k<k^{*}$, free entry leads to excessive numbers of firms for both consumer and total welfare, whereas when $k>k^{*}$, entry is deficient for consumer welfare and, for sufficiently large $k$, also for total welfare.

Our result on how entry affects consumer welfare, while unconventional, is quite natural for search markets, with the following intuition: when $k$ (or $\left.t_{f}\right)$ is high, the expected number of entrants is small but the marginal entrant's quality is high, and hence for a marginal decrease in $k$ (or $t_{f}$ ), the positive variety effect outweighs the negative quality effect. Conversely, when $k$ is low, a large number of firms enter but the marginal entrant's quality is low, and hence for a marginal increase in $k$, the positive quality effect dominates the negative variety effect. ${ }^{5}$

To investigate the robustness of our results and to gain additional insights, we further study a variant of the main model, in which a consumer's value for each match is an independent random draw. The matched sellers of any consumer are then horizontally differentiated, as in Wolinsky (1986); ${ }^{6}$ and everything else is the same as in the main

\footnotetext{
${ }^{5}$ In search markets, more sellers can cause price to rise (e.g., Satterthwaite, 1979; Stahl, 1989), to fall (e.g., Wolinsky, 1986), or to either increase, decrease, or unchange (Janssen and Moraga-González, 2004). Our model thus provides a useful baseline case, making it transparent that the mechanism through which entry affects consumer welfare in our setting differs from the usual price effect.

${ }^{6}$ Search models with horizontally differentiated sellers following Wolinsky (1986) include, for example,
} 
model. In addition to the variety and quality effects, entry then also has a price effect, as equilibrium price now varies with the number of sellers in the market. ${ }^{7}$ A finding here, possibly of independent interest, is that equilibrium market price decreases in the expected quality of sellers in the market. The effect of entry on consumer welfare is more complicated in this setting, due to the additional price effect. For the numerical examples we have considered, nevertheless, consumer welfare remains an inverted-U function of entry cost, and the excessive or deficient entry results under free entry also continue to hold.

In search markets, therefore, it will not be unusual for entry restrictions to benefit consumers. This can shed light on many business practices. Consider, for instance, the market of apps for iphones and ipads. Apple clearly has the incentive to increase consumer surplus in this market, which would boost its profits from the sale of iphones and ipads. Whereas more entrants of app developers will offer users more product varieties, the entry of low quality sellers can reduce search efficiency and make it harder for consumers to find a desired app. Apple appears to balance this trade off by creating substantial entry barriers: it charges a fixed fee to each entrant (\$99/year), and the entrant's product needs to go through a stringent review process. Only after the product is approved by Apple can it be offered for sale to consumers. In addition to entry barriers created by private entities (as we shall discuss further in the concluding section), government policies can also limit entry. A minimum quality requirement, for example, can positively impact both consumer and total welfare by raising the quality of the marginal entrant. A license fee that acts as a transfer payment, which may be easier to implement, can have the same beneficial effects. On the other hand, an entry barrier that adds to physical cost of entry (such as transaction cost) might benefit consumers but reduce total welfare.

In the rest of the paper, we describe our model in Section 2, and characterize its equilibrium in Section 3. In Section 4, we study how the equilibrium (expected) number of Anderson and Renault (1999), Armstrong, Vickers and Zhou (2009), Hann and Moraga-González (2011), and Bar-Isaac, Caruana, and Cuñat, 2012).

${ }^{7}$ If the value of a consumer's matched seller is low, the consumer will now have the incentive to search more, to possibly find another match with a higher value. As a result, the market price will depend on the number of sellers. 
entrants, or entry cost, affects consumer and total welfare, and we also compare the free entry outcomes with those maximizing consumer welfare or total welfare. After analyzing the variant of the main model in Section 5, we conclude in Section 6. Some technical proofs are gathered in the Appendix.

\section{THE MODEL}

The market contains a unit mass of consumers, each demanding one unit of a product. There are $N \geq 2$ potential entrants who can choose to become active sellers, and the entry cost for each seller is $k>0$. The production cost of each seller is normalized to zero. Each consumer is ex ante uncertain about whether a particular firm offers a product that she desires and how much she is willing to pay for such a product. Specifically, with probability $\beta_{i}$, potential entrant $i$ 's product, $i=1,2, \ldots N$, meets a consumer's need (or has high value to the consumer). The consumer derives utility $u$ from consuming the product of all her matched sellers, and $u$ is an independent draw from distribution $F$ with density $f$ on support $[\underline{u}, \bar{u}]$, where $\bar{u}>\underline{u} \geq 0$. With probability $1-\beta_{i}$, $i$ 's product does not meet the consumer's need, in which case the consumer utility from the product is normalized to zero. Thus, we consider $\beta_{i}$ as a measure of $i^{\prime} s$ quality. ${ }^{8}$ Potential sellers differ in their quality. In particular, we assume that $\beta_{i}$ draws from cumulative distribution function $G$ with density function $g>0$ on support $[0,1]$. Our formulation is a tractable way of modeling the idea that firms differ both vertically and horizontally, with a higher $\beta_{i}$ corresponding to a higher quality, and firms would only differ horizontally if all $\beta_{i}$ were identical.

The timing of the model is as follows. First, $\beta_{i}$ is realized and is known privately by $i$. Second, potential entrants simultaneously choose either to enter the market or to stay out. Third, the market structure is determined, with $n$ entrants as sellers. Although $n=0$ is always a possibility, our analysis will focus on situations where $n \geq 1$, and we assume that $k$ is relatively small so that a potential entrant with a sufficiently high $\beta_{i}$ will enter the

\footnotetext{
${ }^{8}$ In fact, the expected value of seller $i^{\prime} s$ product to a consumer is simply $\beta_{i} \int_{\underline{u}}^{\bar{u}} u d F(u)$, which increases in $\beta_{i}$.
} 
market. Fourth, sellers simultaneously and independently set their prices, after which each consumer, without knowing whether any particular seller is a match, her value $u$ if there is a match, and the seller's price, chooses whether and how to conduct sequential search. Each search will enable the consumer to discover the aforementioned information from a seller, with search cost $s$. We study symmetric perfect Bayesian equilibrium of this game.

Throughout the paper, we maintain the assumption that $G$ and $F$ satisfy the monotonic hazard-rate condition:

$$
\frac{d\left(\frac{g(\beta)}{1-G(\beta)}\right)}{d \beta} \geq 0 ; \quad \frac{d\left(\frac{f(u)}{1-F(u)}\right)}{d u} \geq 0 .
$$

Let

$$
p^{o}=\arg \max _{p}\{p[1-F(p)]\} ; \quad \pi^{o}=p^{o}\left[1-F\left(p^{o}\right)\right] .
$$

Then, from (1), $p^{o}$ uniquely exists. Condition (1) will facilitate our analysis both in the main model and in its variant.

\section{MARKET EQUILIBRIUM}

Suppose for a moment that, given $k$, a potential entrant will enter the market if and only if its quality exceeds some threshold $t$. We first study equilibrium for any given threshold $t$. We then show that in equilibrium the expected profit of potential entrant $i$ indeed increases in $\beta_{i}$, thereby confirming the optimality of the threshold-based entry strategy for each potential entrant. The equilibrium threshold $t_{f}$ is then determined, which is shown to increase in $k$.

For any given $t$, the expected match probability of an entrant is

$$
\gamma \equiv \gamma(t)=\frac{\int_{t}^{1} x g(x) d x}{1-G(t)}
$$

where $\gamma>t$ for all $t \in[0,1)$ since $\int_{t}^{1} x g(x) d x>t[1-G(t)]$.

First, consider the sellers' price strategy and consumers' search strategy. If there is only one seller $(n=1)$, its equilibrium price will be $p^{o}$, and consumers will search if

$$
\gamma \int_{p^{o}}^{\bar{u}}\left(u-p^{o}\right) f(u) d u-s \geq 0 .
$$


Condition (3) is satisfied if $s$ is not too large, which we assume throughout the paper.

With $n \geq 2$ sellers, from standard arguments (e.g., Diamond, 1971; Chen and He, 2011), there is a unique equilibrium where each seller sets $p=p^{o}$, each consumer will search sequentially and will purchase from the first match, provided that $u \geq p^{o}$. The consumer will exit the market without purchase if $u<p^{o}$ or if she has searched all $n$ sellers without finding a match.

Thus, in equilibrium, seller $i^{\prime} s$ expected profit for any given $t$ is

$$
\pi_{n}\left(\beta_{i}\right)=\beta_{i} \pi^{o} \phi_{n}
$$

where

$$
\phi_{n}=\frac{1}{n} \sum_{j=0}^{n-1}(1-\gamma)^{j}=\frac{1-(1-\gamma)^{n}}{n \gamma}
$$

is the number of consumers who come to seller $i$ for the first time after sampling $j \in$ $\{0,1, \ldots, n-1\}$ other sellers.

We next determine the endogenous number of sellers. Consider a potential seller's entry decision. From (4), a seller's expected profit, when there are $n$ entrants, is increasing in $\beta_{i}$. To determine the equilibrium $t$, we consider the decision of $i$ with $\beta_{i}$. The post-entry expected profit for $i$ is

$$
E\left(\pi \mid \beta_{i}\right)=\sum_{n=1}^{N} \delta_{n}(t) \pi_{n}\left(\beta_{i}\right),
$$

where

$$
\delta_{n}(t)=\left(\begin{array}{l}
N-1 \\
n-1
\end{array}\right)[1-G(t)]^{n-1} G(t)^{N-n}
$$

is the probability that $n-1$ other potential entrants enter and $\pi_{n}\left(\beta_{i}\right)$ is the expected profit for $i$ if it chooses entry simultaneously as the $n-1$ others. Our analysis will utilize Lemma 1 below, which states that (i) an increase in the marginal entrant's quality will raise the average quality of all entrants in the market, but (ii) the marginal increases relatively more than the average. Part (i) is straightforward, and while (ii) is also intuitive, it relies on the hazard-rate condition (1). 
Lemma 1 For all $t \in[0,1)$ :

$$
\text { (i) } \frac{d \gamma}{d t}=\frac{g(t)}{1-G(t)}(\gamma-t)>0 ; \quad(i i) \frac{d(t / \gamma(t))}{d t}=\frac{\gamma-\frac{g(t) t(\gamma-t)}{1-G(t)}}{\gamma^{2}}>0 .
$$

By Lemma 1, the proof of which is contained in the Appendix,

$$
\pi_{n}(t)=\pi^{o} \frac{t}{\gamma} \frac{1-(1-\gamma)^{n}}{n}
$$

increases in $t$. That is, given $n$, the expected profit for the marginal entrant is higher if it has a higher quality. It can also be verified that $\pi_{n}(t)$ decreases in $n$. Lemma 2 , which is also proved in the Appendix, establishes that the expected post-entry profit for the marginal entrant is increasing in its quality:

Lemma $2 E(\pi \mid t)$ increases in $t$.

Notice that the marginal entrant will earn zero if it has $\beta_{i}=0$, and will earn $\pi^{o}$ if it has $\beta_{i}=1$. Therefore, for any given $k \in\left[0, \pi^{o}\right)$, there exists a unique threshold $t_{f} \equiv t_{f}(k) \in$ $[0,1)$ that satisfies

$$
E\left(\pi \mid t_{f}\right)=k
$$

and $t_{f}=t_{f}(k)$ increases in $k$, with $t_{f}=0$ for $k=0$ and $t_{f} \rightarrow 1$ as $k \rightarrow \pi^{o}$. We have thus shown that there exists a symmetric equilibrium where each potential entrant will enter if and only if its quality reaches the threshold $t_{f}$, and $t_{f}$ monotonically increases in $k$. Moreover, it is straightforward to check that there can be no other symmetric equilibrium.

Summarizing the above discussion, we have:

Proposition 1 For any given $k \in\left(0, \pi^{o}\right)$, there exists a unique symmetric equilibrium where: (i) potential entrant $i, i=1,2, \ldots, N$, will enter the market if and only if $\beta_{i} \geq t_{f}$, with $t_{f} \in(0,1)$, defined in (10), being an increasing function of $k$, and each seller will charge price $p^{o}$; (ii) each consumer will search sequentially in random order, purchase from the first match if $u \geq p^{o}$, and make no purchase if either she finds no match or $u<p^{o}$. 


\section{WELFARE ANALYSIS}

In our model, the number of entrants $(n)$ is uncertain, depending on the number of potential entrants $(N)$, the realizations of $\beta_{i}$, and entry cost $(k)$. Hence a proper measure of entry is the expected number of entrants, which is determined by $t$, the minimum possible quality of actual entrants. A lower $t$ corresponds to a higher expected number of sellers in the market. In equilibrium, through the dependence of $t_{f}$ on $k$, the expected number of sellers in turn will be determined by $k$.

We are interested in two related questions on consumer and total welfare. First, how will an exogenous change in entry conditions, such as entry cost, affect welfare in the free entry equilibrium? Second, given the entry conditions, how will the expected number of entrants under free entry compare to those that maximize consumer or total welfare?

\subsection{Consumer Welfare}

For a given $t$, consumer welfare, measured by expected aggregate consumer surplus (net of search cost), is

$$
V=\sum_{n=1}^{N} \lambda_{n}(t) V_{n}\left(\gamma, p^{o}\right)
$$

where

$$
\lambda_{n}(t)=\left(\begin{array}{l}
N \\
n
\end{array}\right)[1-G(t)]^{n} G(t)^{N-n}
$$

is the probability that exactly $n$ sellers have entered, and

$$
V_{n}\left(\gamma, p^{o}\right)=\sum_{i=1}^{n}(1-\gamma)^{i-1} \gamma \int_{p^{o}}^{1}\left(u-p^{o}\right) f(u) d u-\sum_{i=1}^{n}(1-\gamma)^{i-1} \gamma i s-(1-\gamma)^{n} n s
$$

is the consumer welfare with $n \geq 1$ sellers when their expected quality is $\gamma$. In $V_{n}$ above, the first term is the (weighted) sum of benefit when a consumer has searched and purchased from the $i^{\text {th }}$ seller, while the second and the third terms are the expected search cost when the consumer ends up with and without purchase, respectively. We define:

$$
\Phi=\int_{p^{o}}^{\bar{u}}\left(u-p^{o}\right) f(u) d u ; \quad M(t)=1-\gamma[1-G(t)]
$$


where $\Phi$ is a consumer's expected surplus from a match, and $M(t)$ indicates the probability that a potential entrant will not be a match when the entry threshold is $t$.

Lemma 3 Consumer welfare $V \equiv V(t)$ can be expressed as:

$$
V=\left[1-M(t)^{N}\right]\left(\Phi-\frac{s}{\gamma}\right)
$$

Proof. From (13), consumer surplus when $n$ sellers are active is

$$
\begin{aligned}
V_{n} & =\left[\frac{1-(1-\gamma)^{n}}{\gamma}\right] \gamma \int_{p^{o}}^{1}\left(u-p^{o}\right) f(u) d u-\left[\frac{1-(1-\gamma)^{n}}{\gamma^{2}}-\frac{n(1-\gamma)^{n}}{\gamma}\right] \gamma s-(1-\gamma)^{n} n s \\
& =\left[\frac{1-(1-\gamma)^{n}}{\gamma}\right]\left[\gamma \int_{p^{o}}^{1}\left(u-p^{o}\right) f(u) d u-s\right]=\left[1-(1-\gamma)^{n}\right]\left(\Phi-\frac{s}{\gamma}\right)
\end{aligned}
$$

where we have used the fact that $\sum_{i=1}^{n} x^{i-1} i=\frac{1-x^{n}}{(1-x)^{2}}-\frac{n x^{n}}{1-x}$. Hence, from (11), consumer welfare is

$$
\begin{aligned}
V & =\left(\Phi-\frac{s}{\gamma}\right)\left(\sum_{n=1}^{N}\left(\begin{array}{l}
N \\
n
\end{array}\right)[1-G(t)]^{n} G(t)^{N-n}-\sum_{n=1}^{N}\left(\begin{array}{l}
N \\
n
\end{array}\right)[1-G(t)]^{n} G(t)^{N-n}(1-\gamma)^{n}\right) \\
& =\left\{1-[1-\gamma(1-G(t))]^{N}\right\}\left(\Phi-\frac{s}{\gamma}\right)=\left[1-M(t)^{N}\right]\left(\Phi-\frac{s}{\gamma}\right) .
\end{aligned}
$$

Equation (15) has an intuitive interpretation. The probability that a consumer will (eventually) find a match is $1-M(t)^{N}$. Since $\Phi$ is the expected surplus to a consumer from a match and $s / \gamma$ is the search cost adjusted by the expected match probability per seller, $\Phi-\frac{s}{\gamma}$ reflects the expected net benefit from a search that yields a match. With a unit mass of consumers, consumer welfare is the consumer's expected net benefit from the entry of firms under threshold $t$.

Notice that given the distribution of $u$, search cost $s$, and the number of potential entrants $N, V$ is entirely determined by $t$ through $\gamma=\gamma(t)$ and $M(t)$. Totally differentiating (15) with respect to $t$ and collecting terms yield

$$
\frac{d V}{d t}=\underbrace{-N M(t)^{N-1}(\gamma \Phi-s) g(t)}_{\text {variety effect }}+\underbrace{\frac{1}{\gamma}\left[\frac{1-M(t)^{N}}{\gamma} s+N M(t)^{N-1}(\gamma \Phi-s)(1-G(t))\right] \frac{d \gamma}{d t}}_{\text {quality effect }} .
$$


Thus, the impact of increased entry (i.e., a decrease in $t$ ) on consumer welfare can be decomposed into two parts: a variety effect and a quality effect. Recall from (3) that $\gamma \Phi>s$, and hence more entry has a positive variety effect: a decrease in $t$ raises the expected number of entrants, providing consumers with more search opportunities. However, from (8), $\frac{d \gamma}{d t}>0$, and hence more entry has a negative quality effect: a decrease in $t$ reduces the average match probability of sellers in the market, lowering consumer search efficiency. The change in consumer welfare depends on the balance of these two opposing effects. Since $t_{f}(k)$ is monotonically increasing, a reduction in $k$ has the same two effects as a reduction in $t_{f}$ in equilibrium.

Define $V_{f} \equiv V\left(t_{f}\right)$ as the consumer welfare in the free-entry equilibrium. The following result states that $V_{f}$ in fact has an inverted-U relationship with entry cost. The proof first establishes that $V(t)$ has an inverted-U shape, and then uses the fact that $t_{f}$ monotonically increases in $k$.

Proposition 2 In equilibrium, consumer welfare is an inverted- $U$ function of entry cost $k$, first increasing and then decreasing, maximized at some $k^{*} \in\left(0, \pi^{o}\right)$.

Proof. From (14) and by Lemma 1,

$$
\frac{d M(t)}{d t}=-\frac{d \gamma}{d t}[1-G(t)]+\gamma g(t)=g(t) t
$$

Noticing $\frac{1}{1-G(t)}=\frac{\gamma}{1-M(t)}$, we have

$$
\begin{aligned}
\frac{d V}{d t} & =\left[1-M(t)^{N}\right] \frac{s}{\gamma^{2}} \frac{d \gamma}{d t}-N M(t)^{N-1} \frac{d M}{d t}\left(\Phi-\frac{s}{\gamma}\right) \\
& =\left[1-M(t)^{N}\right]\left(\frac{s}{\gamma^{2}}\right) \frac{g(t)}{1-G(t)}(\gamma-t)-N M(t)^{N-1} g(t) t\left(\Phi-\frac{s}{\gamma}\right) \\
& =g(t)\left[\frac{1-M(t)^{N}}{1-M(t)} \frac{s}{\gamma}(\gamma-t)-N M(t)^{N-1} t\left(\Phi-\frac{s}{\gamma}\right)\right] .
\end{aligned}
$$

Therefore, for $t \in(0,1), \frac{d V}{d t}=0$ if

$$
\frac{t}{\gamma}=\frac{1}{1+N M(t)^{N-1} \frac{1-M(t)}{1-M(t)^{N}}\left(\frac{\gamma \Phi-s}{s}\right)} .
$$


If $t=0$, the LHS of (19) < the RHS of (19); if $t \rightarrow 1$, the LHS of (19) $>$ the RHS of (19). Furthermore, from Lemma 1 , the LHS of (19) monotonically increases in $t$. Since $\frac{d M(t)}{d t} \geq 0$, $\frac{d \gamma}{d t} \geq 0$, and

$\frac{d\left(M^{N-1} \frac{1-M}{1-M^{N}}\right)}{d M}=\frac{M^{N-2}}{\left(1-M^{N}\right)^{2}}\left(N-N M+M^{N}-1\right)=\frac{M^{N-2}}{\left(1-M^{N}\right)^{2}}(1-M)\left(N-\sum_{j=0}^{N-1} M^{j}\right) \geq 0$,

the RHS of (19) decreases in $t$. Therefore, there exists a unique $t^{*} \in(0,1)$ that solves $(19)$, with $\frac{d V}{d t}>0$ if $t<t^{*}$ and $\frac{d V}{d t}<0$ if $t>t^{*}$. Finally, since $t_{f}=t(k)$ is monotonically increasing and $\frac{d V_{f}}{d k}=\frac{d V_{f}}{d t_{f}} t_{f}^{\prime}(k)$, it follows that $V_{f}$ first increases and then decreases in $k$, maximized at some $k^{*} \in\left(0, \pi^{o}\right)$.

As $t$, or entry cost $k$, decreases, more potential entrants choose to enter the market, but the marginal entrant has a lower quality. Holding other things constant, an increase in the number of sellers in the market is beneficial to consumers (the variety effect), because more sellers increase the chance that the consumer will eventually find a match; but the addition of lower-quality sellers reduces the average seller quality, which harms consumers because they expect to search more sellers before possibly finding a match (the quality effect). When $k$ is high, the number of sellers is low. In this case, the positive variety effect from a decrease in $k$ dominates, both because of the additional search opportunities and of the relatively high qualities of the marginal entrants. However, when $k$ is relatively low, a further decrease in $k$ attracts sellers with low qualities, while the benefit from more search opportunities is also small; consequently, the quality effect dominates and consumer welfare decreases.

Since the entry threshold associated with the maximum consumer welfare is $t^{*}=t_{f}\left(k^{*}\right)$, Proposition 2 immediately implies that, from the consumer welfare perspective, free entry is excessive under low entry cost but deficient under high entry cost. Or, more precisely:

Corollary 1 Relative to what maximizes consumer welfare, the expected number of entrants under free entry is too high when $k<k^{*}$ and too low when $k>k^{*}$.

We also have the following result concerning the entry cost that maximizes consumer welfare, $k^{*}$. 
Corollary $2 k^{*}$, or $t^{*}$, increases in search cost (s) and in the number of potential entrants $(N)$.

Proof. Since $t^{*}=t\left(k^{*}\right)$, it suffices to show that $t^{*}$ increases in $s$ and in $N$. Since LHS of (19) increases in $t$ and is independent of $s$ while RHS decreases in $t$ and increases in $s$, $t^{*}$ increases in $s$. Moreover, since $M<1, d\left[N \ln M-M^{N}+1\right] / d M=\frac{N}{M}-N M^{N-1}>0$, and $N \ln 1-1^{N}+1=0$, we have

$$
d\left(N M^{N-1} \frac{1-M}{1-M^{N}}\right) / d N=\frac{M^{N-1}}{\left(1-M^{N}\right)^{2}}(1-M)\left(N \ln M-M^{N}+1\right)<0 .
$$

Therefore, $t^{*}$ increases in $N$.

Thus, the entry cost (or the quality threshold) that maximizes consumer welfare increases in search cost and in the number of potential entrants. Intuitively, with a high search cost, it is more costly for consumers to search more varieties. It follows that fewer sellers with higher quality tend to be better for consumers, and hence $k^{*}$ (or $t^{*}$ ) is higher. Also, when the number of potential sellers is high, the variety effect is less significant because for a given $k$ the expected number of entrants is large, and hence an increase in $t$ tends to be more beneficial to consumers. Therefore, $t^{*}$ also increases in the number of potential entrants.

\subsection{Total Welfare}

We next consider total welfare. For given $k$ and $t$, the (expected) industry profit is

$$
\Pi=\sum_{n=0}^{N} \lambda_{n}(t) n\left[\pi_{n}(\gamma)-k\right],
$$

where $\pi_{n}(\gamma)-k$ is the expected profit for a seller of quality $\gamma$ in a market with $n$ sellers. We have:

Lemma 4 For any given $t$, industry profit is

$$
\Pi(t)=\pi^{o}\left[1-M(t)^{N}\right]-k N[1-G(t)],
$$

and the free-entry equilibrium industry profit is

$$
\Pi_{f}=\left(1-\frac{t_{f}}{\gamma}\right)\left[1-M\left(t_{f}\right)^{N}\right] \pi^{o} .
$$


Proof. Given that there are $n$ sellers and each seller's expected match probability is $\gamma$, the expected industry profit is

$$
n \pi_{n}(\gamma)=\pi^{o}\left[1-(1-\gamma)^{n}\right]
$$

Then, from (20) and (12),

$$
\begin{aligned}
\Pi(t) & =\pi^{o} \sum_{n=0}^{N} \lambda_{n}(t)\left[1-(1-\gamma)^{n}\right]-k \sum_{n=0}^{N} \lambda_{n}(t) n \\
& =\pi^{o}\left\{1-\sum_{n=0}^{N} \frac{N !}{n !(N-n) !}[1-G(t)]^{n} G(t)^{N-n}(1-\gamma)^{n}\right\}-k N[1-G(t)] \\
& =\pi^{o}\left[1-M(t)^{N}\right]-k N[1-G(t)] .
\end{aligned}
$$

Moreover, from (10), under free-entry $t_{f}$ satisfies

$$
\begin{aligned}
k & =\frac{t \pi^{o}}{\gamma} \sum_{n=1}^{N}\left(\begin{array}{l}
N-1 \\
n-1
\end{array}\right)[1-G(t)]^{n-1} G(t)^{N-n} \frac{1-(1-\gamma)^{n}}{n} \\
& =\frac{t \pi^{o}}{\gamma}\left\{\sum_{n=1}^{N} \frac{(N-1) !}{n !(N-n) !}[1-G(t)]^{n-1} G(t)^{N-n}-\sum_{n=1}^{N} \frac{(N-1) !}{n !(N-n) !}[1-G(t)]^{n-1} G(t)^{N-n}(1-\gamma)^{n}\right\} \\
& =\frac{t \pi^{o}}{\gamma}\left[\frac{1-G(t)^{N}}{N[1-G(t)]}-\frac{M(t)^{N}-G(t)^{N}}{N[1-G(t)]}\right]=\frac{t}{\gamma} \pi^{o}\left[\frac{1-M(t)^{N}}{N[1-G(t)]}\right] .
\end{aligned}
$$

Therefore, the free-entry equilibrium industry profit is

$$
\begin{aligned}
\Pi_{f} & =\sum_{n=0}^{N} \lambda_{n}\left(t_{f}\right) n\left[\pi_{n}(\gamma)-k\right]=\pi^{o} \sum_{n=1}^{N} \lambda_{n}\left(t_{f}\right)\left[1-(1-\gamma)^{n}\right]-N\left[1-G\left(t_{f}\right)\right] k \\
& =\pi^{o}\left[1-M\left(t_{f}\right)^{N}\right]-\frac{t_{f}}{\gamma}\left[1-M\left(t_{f}\right)^{N}\right] \pi^{o} \\
& =\left(1-\frac{t_{f}}{\gamma}\right)\left[1-M\left(t_{f}\right)^{N}\right] \pi^{o} .
\end{aligned}
$$

Notice that $\left[1-M\left(t_{f}\right)^{N}\right] \pi^{o}$ is the expected industry revenue when at least one seller's product matches a consumer's need. Since the marginal entrant with $t_{f}$ earns zero profit, $1-\frac{t_{f}}{\gamma}$ reflects the expected profit margin of each entrant.

From (15) and (22), total welfare at the free entry equilibrium is

$$
W_{f}=\left[1-M\left(t_{f}\right)^{N}\right]\left[\left(\Phi-\frac{s}{\gamma}\right)+\left(1-\frac{t_{f}}{\gamma}\right) \pi^{o}\right] .
$$


Proposition 3 In equilibrium: (i) industry profit decreases in $k$; (ii) social welfare decreases in $k$ when $s$ is sufficiently small or $k$ is sufficiently high.

Proof. From (22) and (23), since $k$ affects $\Pi_{f}$ and $W_{f}$ only through $t_{f}$, and since $t_{f}$ increases in $k$, it suffices to show that the stated relationships for $k$ hold for $t_{f}$. (i) Recall from (8) and (17) that $\frac{d(t / \gamma)}{d t}>0$ and $\frac{d M}{d t} \geq 0$. Thus $\frac{d \Pi_{f}}{d t_{f}}<0$. (ii) From Proposition 1 , consumer welfare decreases in $t$ when $t$ is high. Thus, since $\frac{d \Pi_{f}}{d t_{f}}<0, W_{f}=V_{f}+\Pi_{f}$ must decrease in $t_{f}$ when $t_{f}$ is sufficiently high. Furthermore,

$$
\begin{aligned}
\frac{d W_{f}}{d t_{f}}= & -N M\left(t_{f}\right)^{N-1} g\left(t_{f}\right) t_{f}\left[\left(\Phi-\frac{s}{\gamma}\right)+\left(1-\frac{t_{f}}{\gamma}\right) \pi^{o}\right] \\
& +\left[1-M\left(t_{f}\right)^{N}\right]\left[\frac{s}{\gamma^{2}} \frac{d \gamma}{d t_{f}}-\frac{d\left(t_{f} / \gamma\right)}{d t_{f}} \pi^{o}\right] .
\end{aligned}
$$

Recall that $\frac{d\left(t_{f} / \gamma\right)}{d t_{f}}>0$. Hence, $\frac{d W_{f}}{d t_{f}}<0$ if $s \rightarrow 0$.

A marginal increase in entry cost raises $t_{f}$, which reduces the expected number of sellers, and, hence, the probability of sales. Additionally, a higher entry cost reduces an inframarginal seller's profit margin. Consequently, industry profit is reduced with a higher entry cost. On total welfare, a higher $k$ will increase consumer welfare by raising $t_{f}$ when $k<k^{*}$, which can potentially outweigh the profit effect. But when $k$ is large, profit and consumer welfare move in the same direction, and hence $W$ is lower with an even higher $k$. Also, when $s$ is small, the low search cost can largely offset the reduction in sellers' quality to provide search incentives, so that the profit change will dominate and hence an increase in entry cost will lower total welfare.

Example 1 below illustrates how the equilibrium consumer welfare, industry profit and total welfare vary with entry cost $k$.

Example 1 Suppose that $N=3, s=0.05$, with $\beta_{i}$ and $u$ being uniformly distributed on $[0,1]$. Then, from (2) and (14), $\gamma=\frac{1+t}{2}, M=\frac{1+t^{2}}{2}, \Phi=\frac{1}{8}$ and $\pi^{o}=\frac{1}{4}$. From (6), $t_{f}$ solves $E(\pi \mid t)=\frac{1}{48} t\left(4 t^{2}+t^{4}+7\right)=k$. From $(15), V_{f}=\frac{1}{320}\left(5 t_{f}+1\right)\left(1-t_{f}\right)\left(4 t_{f}^{2}+t_{f}^{4}+7\right), t^{*}=$ 0.497 , and $k^{*}=0.083$. Moreover, from (22), $\Pi_{f}=\frac{1}{32}\left(1-t_{f}\right)^{2}\left(4 t_{f}^{2}+t_{f}^{4}+7\right)$, and thus 
$W_{f}=\frac{1}{320}\left(11-5 t_{f}\right)\left(1-t_{f}\right)\left(4 t_{f}^{2}+t_{f}^{4}+7\right)$. In Figure 1, consumer welfare is the invertedU curve (solid curve), while both industry profit (dash curve) and total welfare (dot curve) decrease with $k$.

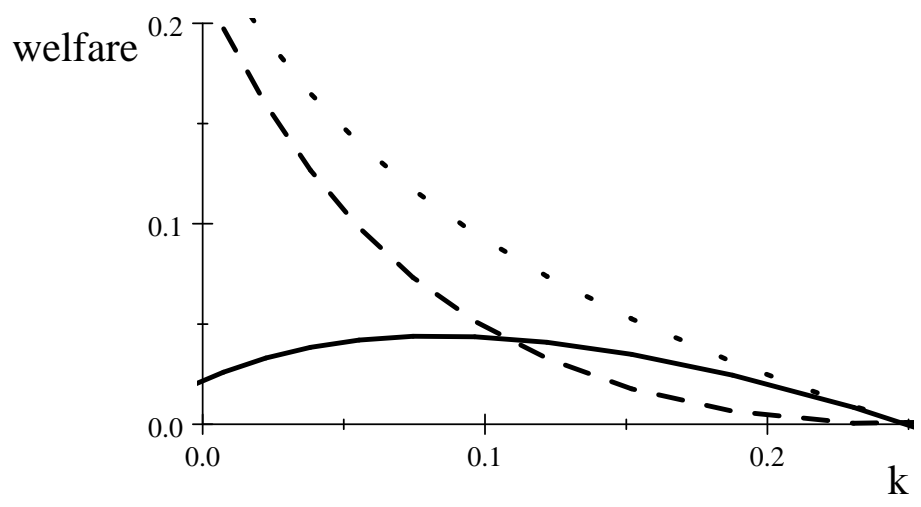

Figure 1

Now consider the socially optimal $t$, denoted as $t^{o} \equiv t^{o}(k)$, for which we do not impose the free-entry condition $E(\pi \mid t)=k$. From (21) and (15), for any given $t$, total welfare is given by

$$
W(t)=\left(\Phi-\frac{s}{\gamma}+\pi^{o}\right)\left[1-M(t)^{N}\right]-k N[1-G(t)]
$$

Thus,

$$
\frac{d W}{d t}=\frac{s}{\gamma^{2}} \frac{d \gamma}{d t}\left[1-M(t)^{N}\right]-\left(\Phi-\frac{s}{\gamma}+\pi^{o}\right) N M(t)^{N-1} \operatorname{tg}(t)+k N g(t) .
$$

At the free entry equilibrium, since the marginal entrant has zero net profit due to $E\left(\pi \mid t_{f}\right)=k$, the marginal entrant must reduce industry profit due to the business-stealing effect. From Proposition 2, for $t_{f}<t^{*} \equiv t_{f}\left(k^{*}\right)$, free entry is excessive for consumer welfare. Therefore, when $k \leq k^{*}$ (or $t_{f} \leq t^{*}$ ), free entry must be socially excessive, with $t^{o}>t_{f}$. When $k>k^{*}$, entry is deficient for consumer welfare, but it can still be socially excessive when the negative profit effect is considered. However, when $k$ is large, the profit effect is small relative to the effect on consumers, and entry is socially deficient, as we establish in the result below. 
Proposition 4 Free entry is socially excessive (i.e., $t^{o}>t_{f}$ ) when $k \leq k^{*}$, and it is socially deficient (i.e., $t^{o}<t_{f}$ ) when $k$ is sufficiently large (but still smaller than $\pi^{o}$ ).

Proof. We have already argued $t^{o}>t_{f}$ when $k \leq k^{*}$. It remains to show $t^{o}<t_{f}$ when $k(<$ $\left.\pi^{o}\right)$ is sufficiently large. From the proof of Lemma 4 , any $t \geq t_{f}$ satisfies $k \leq \frac{t}{\gamma} \pi^{o}\left[\frac{1-M(t)^{N}}{N[1-G(t)]}\right]$. Substituting this into (25), we have

$$
\left.\frac{d W}{d t}\right|_{t \geq t_{f}} \leq\left[\frac{1-M(t)^{N}}{1-G(t)}\left(\frac{s(\gamma-t)}{\gamma^{2}}+\frac{t}{\gamma} \pi^{o}\right)-\left(\Phi-\frac{s}{\gamma}+\pi^{o}\right) N M(t)^{N-1} t\right] g(t) .
$$

Thus, when $k \rightarrow \pi^{o}, t \rightarrow 1, \gamma \rightarrow 1, M(t) \rightarrow 1$ and, from $(17), \lim _{t \rightarrow 1} \frac{1-M(t)^{N}}{1-G(t)}=$ $\lim _{t \rightarrow 1} \frac{-N \cdot M(t)^{N-1} g(t) t}{-g(t)}=N$. Hence, the right-hand side of the above inequality approaches

$$
-(\Phi-s) N g(1)<0
$$

Therefore, when $k$ is sufficiently large (but still smaller than $\pi^{o}$ ), $\left.\frac{d W}{d t}\right|_{t \geq t_{f}}<0$, so that free entry is socially deficient (i.e., $t^{o}<t_{f}$ ).

\subsection{Effects of the Number of Potential Entrants $(N)$}

Our proceeding analysis has assumed that the number of potential entrants, $N$, is given. We now discuss the welfare effects of $N$. Potentially, $N$ can also be affected by private arrangements and government policies.

A change in $N$ affects $V$ directly and also indirectly by impacting $t$. Holding $t$ constant, an increase in $N$ increases $V$; but the indirect effect of $N$ through $t$ is more complicated, partly because $V$ is non-monotonic in $t$. While a general analysis of the effects of $N$ on consumer and total welfare is beyond the scope of the paper, numerical analysis of several examples suggest that consumer welfare monotonically increases in $N$ when $N$ is relatively small, whereas industry profit and total welfare may first increase and then decrease in $N$. One example is below:

Example 2 Suppose that $s=0.05$, with $\beta_{i}$ and $u$ being uniformly distributed on $[0,1]$. Then, from (2) and (14), $\gamma=\frac{1+t}{2}, M=\frac{1+t^{2}}{2}, \Phi=\frac{1}{8}$ and $\pi^{o}=\frac{1}{4}$. From (10), the 
equilibrium $t_{f}$ is determined by $k=\frac{t_{f}}{\gamma} \pi^{o} \frac{1-M^{N}}{N\left(1-t_{f}\right)}$. Suppose further that $k=0.03$. Then for each $N$, we can compute $t_{f}$ and substitute $t_{f}$ into $V, \Pi_{f}$ and $W_{f}$. For $N \leq 10$, Figure 2 below illustrates the impact of $N$ on consumer welfare (solid curve), industry profit (dash curve), and total welfare (dot curve).

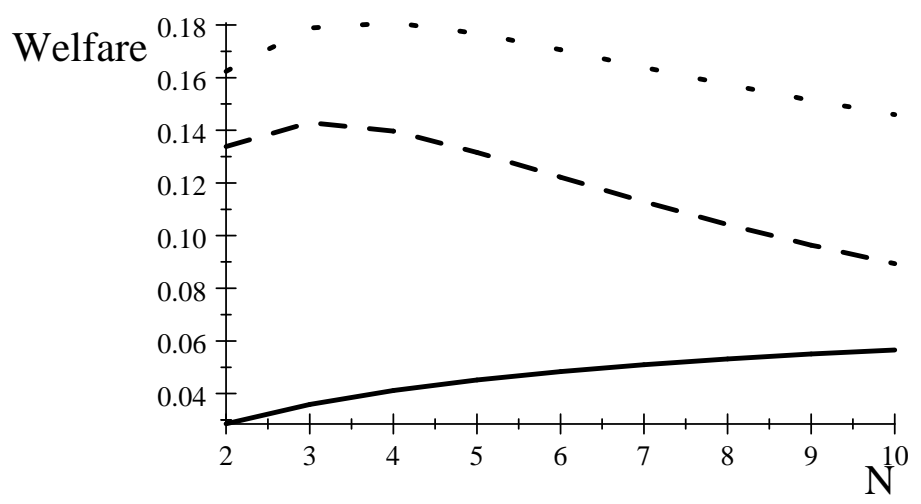

Figure 2

While the relationships illustrated in Figure 2 are based on specific parameter values, they are likely to hold in more general settings, for the following intuition. First, an increase in $N$ leads to more entrants for any given $t$, and hence to lower profits for the marginal entrant. Thus $t_{f}$ increases in $N$. Second, holding other things constant, from (15), an increase in $N$ increases consumer welfare $V$. Moreover, from Proposition 2, $V$ increases in $t$ if $t$ is relatively small. It follows that when $N$ is relatively small, consumer welfare tends to increase in $N$, as in Figure 2. Third, a change in $N$ also affects $\Pi$ and $W$ both directly and indirectly through $t$ : holding other things constant, an increase in $N$ positively affects industry profit and thus total welfare; but the higher $t$ due to a higher $N$ may negatively impact industry profit and total welfare (as in Figure 1). The direct effect or the indirect effect can respectively dominate when $N$ is relatively small or relatively large, as in Example 2.

We summarize the above discussion in the following:

Remark 1 An increase in the number of potential entrants can boost consumer welfare but lower industry profit and total welfare. 


\section{DIFFERENTIATION AMONG MATCHED SELLERS}

So far, we have assumed that a consumer has the same value $(u)$ from all of her matched sellers, even though $u$ is ex ante uncertain to the consumer. As we mentioned earlier, one advantage of this formulation is that equilibrium price will then be invariant to the number of sellers, which substantially simplifies the analysis. We now consider an alternative setting where a consumer has heterogeneous values for sellers who match her need. Specifically, as in Wolinsky (1986), we assume that a consumer's value for each matched seller $i, u_{i}$, is independently drawn from distribution $F$ on support $[0, \bar{u}]$, with density $f .{ }^{9}$ Thus, there is horizontal differentiation among matched sellers. Everything else is the same as in the main model.

A key aspect in which this variant differs from the main model is that entry will now also affect market price. Our analysis in this section proceeds as follows: First, we characterize the equilibrium pricing strategy given the number of active sellers $(n)$ and their average quality $(\gamma)$. Next, we show that the equilibrium market price $\left(p_{n}\right)$ decreases in $\gamma$. This additional price effect introduces a complication to the expected profit for a seller. In particular, unlike in the main model, it is no longer clear that a potential entrant's expected profit will increase in $t$, because a higher $t$, which results in a higher average quality $\gamma$, now also leads to a lower equilibrium price. After presenting the equilibrium analysis for a given $n$, we will turn to numerical analysis to show that the welfare results of the main model still hold under additional functional and parameter restrictions.

Suppose first that there are $n \leq N$ sellers in the market. Following Weitzman (1979) and Wolinsky (1986), consumers' optimal search strategy is to sample sellers sequentially, with reservation value $a(\gamma)$ from matched seller $i$ that satisfies

\footnotetext{
${ }^{9}$ That is, in contrast to our main model in which the values of a consumer's matched sellers are perfectly dependent, this formulation considers the other polar case where these values are independent. More realistically, the values of a consumer's matched sellers may be neither perfectly dependent nor independent; but, like others in the literature, we focus on these two polar cases for analytical tractability.
} 


$$
\gamma \int_{a}^{\bar{u}}\left(u_{i}-a\right) f\left(u_{i}\right) d u_{i}=s
$$

Note that the market is active only when sellers are expected to charge $p_{n} \leq a$. A consumer stops searching when she finds a match with $u_{i} \geq a$; if no such product is found after she searches all sellers, she buys the product from the matched seller with the highest $u_{i} \geq p_{n}$, and she buys nothing if no match is found or if $u_{i}<p_{n}$ for all matches. Since $u_{i}$ is independently and identically distributed for each of a consumer's matched sellers, for convenience we shall drop the subscript $i$ for the rest of the section.

Total differentiation of (26) with respect to $\gamma$ and rearranging terms, we have

$$
\frac{\partial a}{\partial \gamma}=\frac{\int_{a}^{\bar{u}}(u-a) f(u) d u}{\gamma \int_{a}^{\bar{u}} f(u) d u}=\frac{s}{\gamma^{2}[1-F(a)]}>0 .
$$

Hence, $a$ increases with $\gamma$. In words, the benefit of search is larger if the expected quality of sellers is higher. We assume that $s$ is sufficiently small such that consumers will indeed search in equilibrium.

Next, we argue that there exists an equilibrium such that sellers charge the same price despite differences in match probabilities. ${ }^{10}$ If there is only one seller $(n=1)$, then it optimally charges $p_{1}=p^{o}$. So suppose that $n \geq 2$. If other sellers charge $p_{n}$ in equilibrium, given the search strategy by consumers, a seller with $\beta_{i}$ charges $p$ to maximize

$$
\pi_{n i}\left(p, p_{n}\right)=p\left\{\beta_{i}\left[1-F\left(p+a-p_{n}\right)\right] \varphi_{n}+\beta_{i} R_{n}\left(p, p_{n}\right)\right\}
$$

where

$$
\varphi_{n}=\frac{1}{n} \sum_{j=0}^{n-1} \sum_{h=0}^{j}\left(\begin{array}{l}
j \\
h
\end{array}\right)(1-\gamma)^{h}[\gamma F(a)]^{j-h}=\frac{1-[1-\gamma+\gamma F(a)]^{n}}{n \gamma[1-F(a)]}
$$

is the number of consumers who come to seller $i$ for the first time after sampling $j \in$

\footnotetext{
${ }^{10}$ Intuitively, all matched sellers of a consumer are horizontally differentiated as in Wolinsky (1986), and thus the equilibrium has a similar structure.
} 
$\{0,1, \ldots, n-1\}$ other sellers and finding no match or the valuation is below $a$, and

$$
\begin{aligned}
R_{n}\left(p, p_{n}\right) & =\int_{p}^{p+a-p_{n}}\left[\sum_{j=0}^{n-1}\left(\begin{array}{l}
n-1 \\
j
\end{array}\right)(1-\gamma)^{n-1-j}\left[\gamma F\left(u-p+p_{n}\right)\right]^{j}\right] f(u) d u \\
& =\int_{p}^{p+a-p_{n}}\left[1-\gamma+\gamma F\left(u-p+p_{n}\right)\right]^{n-1} f(u) d u
\end{aligned}
$$

is the number of returning consumers who have sampled all sellers and have not found any value above $a$, while seller $i$ is a match that gives the highest valuation. It follows that

$$
R_{n}\left(p_{n}, p_{n}\right)=\int_{p_{n}}^{a}[1-\gamma+\gamma F(u)]^{n-1} f(u) d u
$$

From the first-order condition of (28), at an equilibrium with $p_{i}=p_{n}$ for all $i=1, \ldots, n$, the equilibrium $p_{n}$ satisfies $\left.\pi_{n i}^{\prime} \equiv \frac{\partial \pi_{n i}}{\partial p}\right|_{p=p_{n}}=0$ :

$[1-F(a)] \varphi_{n}+\int_{p_{n}}^{a}[1-\gamma+\gamma F(u)]^{n-1} d F(u)-p_{n}\left\{f(a) \varphi_{n}-\int_{p_{n}}^{a}[1-\gamma+\gamma F(u)]^{n-1} d f(u)\right\}=0$.

If $p_{n}=0$, the LHS of (32) is positive. If $p_{n}=a$, the LHS of (32) becomes $\{[1-F(a)]-a f(a)\} \varphi_{n}$, which is negative because $a-\frac{1-F(a)}{f(a)}>p_{1}-\frac{1-F\left(p_{1}\right)}{f\left(p_{1}\right)}=0$, where the inequality holds due to $p_{1}<a$ and $\frac{1-F(a)}{f(a)}<\frac{1-F\left(p_{1}\right)}{f\left(p_{1}\right)}$. Thus there exists some $p_{n} \in(0, a)$ that solves $(32)$, and $p_{n}$ is given by

$$
p_{n}=\frac{[1-F(a)] \varphi_{n}+\int_{p_{n}}^{a}[1-\gamma+\gamma F(u)]^{n-1} f(u) d u}{f(a) \varphi_{n}-\int_{p_{n}}^{a}[1-\gamma+\gamma F(u)]^{n-1} f^{\prime}(u) d u} .
$$

This is also the unique symmetric price equilibrium with consumer search, as we establish below. ${ }^{11}$ The proof basically involves showing that $\pi_{n i}\left(p_{n}, p_{n}\right)$ is strictly concave in $p_{n}$, which relies on the monotonic hazard rate condition (1).

Lemma 5 For the alternative model where each consumer's value is independent for every match, when there are $n$ sellers whose expected match probability is $\gamma$, there exists a unique symmetric price equilibrium with consumer search, where each seller sets $p_{n}$ and consumers search with reservation value $a(\gamma)$ that satisfies (26).

\footnotetext{
${ }^{11}$ Search models generally also have a trivial equilibrium where firms are expected to and indeed charge very high prices, and no consumer engages in search. As in the literature, we do not consider such trivial cases.
} 
Proof. It suffices to show that the symmetric price equilibrium, defined by (33), is unique, which would be the case if $\pi_{n i}\left(p_{n}, p_{n}\right)$ is a strictly concave function of $p_{n}$, or $\pi_{n i}^{\prime \prime}=-\left[1-\gamma+\gamma F\left(p_{n}\right)\right]^{n-1}\left[f\left(p_{n}\right)+p_{n} f^{\prime}\left(p_{n}\right)\right]-\left\{f(a) \varphi_{n}-\int_{p_{n}}^{a}[1-\gamma+\gamma F(u)]^{n-1} f^{\prime}(u) d u\right\}$ is negative. First, from (29), $\varphi_{n}=\frac{1}{n} \sum_{j=0}^{n-1}[1-\gamma+\gamma F(a)]^{j} \geq[1-\gamma+\gamma F(a)]^{n-1}$. Hence

$$
\begin{aligned}
& \int_{p_{n}}^{a}[1-\gamma+\gamma F(u)]^{n-1} f^{\prime}(u) d u \\
\leq & {[1-\gamma+\gamma F(a)]^{n-1} \int_{p_{n}}^{a} f^{\prime}(u) d u=[1-\gamma+\gamma F(a)]^{n-1}\left[f(a)-f\left(p_{n}\right)\right] \leq f(a) \varphi_{n} . }
\end{aligned}
$$

Therefore, $\pi_{n i}^{\prime \prime}<0$ if $f\left(p_{n}\right)+p_{n} f^{\prime}\left(p_{n}\right) \geq 0$. When $f^{\prime} \geq 0$, clearly $\pi_{n i}^{\prime \prime}<0$.

Next, suppose $f^{\prime}<0$. Notice that $d[f(u) /(1-F(u))] / d u \geq 0$ implies $f^{\prime} \frac{1-F}{f}+f \geq 0$. Thus, if $\frac{1-F\left(p_{n}\right)}{f\left(p_{n}\right)} \geq p_{n}$, we have $f\left(p_{n}\right)+p_{n} f^{\prime}\left(p_{n}\right) \geq f\left(p_{n}\right)+\frac{1-F\left(p_{n}\right)}{f\left(p_{n}\right)} f^{\prime}\left(p_{n}\right) \geq 0$, and hence $\pi_{n i}^{\prime \prime}<0$. Finally, we prove that $f\left(p_{n}\right)+p_{n} f^{\prime}\left(p_{n}\right) \geq 0$ if $\frac{1-F\left(p_{n}\right)}{f\left(p_{n}\right)}<p_{n}$. Suppose, to the contrary, that $f\left(p_{n}\right)+p_{n} f^{\prime}\left(p_{n}\right)<0$. Then $f\left(p_{n}\right)<-p_{n} f^{\prime}\left(p_{n}\right)$. It follows from (32) that $[1-F(a)]-p_{n} f(a)>0$, which implies $p_{n}<\frac{1-F(a)}{f(a)} \leq \frac{1-F\left(p_{n}\right)}{f\left(p_{n}\right)}$, a contradiction.

We next state a result on how equilibrium price may vary with the average quality of sellers in the market and with the number of sellers. The proof for the result is contained in the Appendix.

Proposition 5 For the alternative model where each consumer's value is independent for every match: (i) given the number of sellers ( $n)$, an increase in $\gamma$ leads to a decrease in $p_{n}$; (ii) given $\gamma$, an increase in $n$ leads to a decrease in $p_{n}$.

It may seem surprising that a higher average quality would lead to a lower market price, but in a search market this result is quite natural, for the following reason. An increase in the average quality of sellers in the market induces a higher consumer reservation value in their search decision, because the expected benefit from another search is higher. This forces sellers to lower prices in order to induce consumers to purchase without further search.

We next show that, for a given entry cost, there exists a free-entry equilibrium that is 
similar to the one in the main model, with the marginal entrant's quality, $t_{f}$, now defined by (34) below.

Proposition 6 For the alternative model where each consumer's value is independent for every match, and for any $k \in\left(0, \pi^{o}\right)$, there exists an equilibrium where: (i) potential entrant $i$ will enter the market if and only if $\beta_{i} \geq t_{f}$, each entrant will charge $p_{n}$ as in (33) when there are $n$ entrants, and $t_{f}$ satisfies

$$
\sum_{n=1}^{N} \delta_{n}\left(t_{f}\right) t_{f} p_{n} \frac{1-\left[1-\gamma+\gamma F\left(p_{n}\right)\right]^{n}}{n \gamma}=k
$$

(ii) consumers will search sequentially with reservation value a that satisfies (26).

Proof. For a given $t$ and thus $\gamma$, from (28) and (36), in the symmetric equilibrium with $n$ sellers the profit for seller $i$ is

$$
\begin{aligned}
\pi_{n i} & =p_{n}\left\{\beta_{i}[1-F(a)] \frac{1-[1-\gamma+\gamma F(a)]^{n}}{n \gamma[1-F(a)]}+\beta_{i} \int_{p_{n}}^{a}[1-\gamma+\gamma F(u)]^{n-1} f(u) d u\right\} \\
& =\beta_{i} p_{n} \frac{1-\left[1-\gamma+\gamma F\left(p_{n}\right)\right]^{n}}{n \gamma} .
\end{aligned}
$$

Thus, the expected post-entry profit for entrant $i$ is $E\left(\pi \mid \beta_{i}\right)=\sum_{n=1}^{N} \delta_{n}(t) \pi_{n i}$, which increases in $\beta_{i}$. For the seller with match probability $t$, its expected profit from entry is

$$
E(\pi \mid t)=\sum_{n=1}^{N} \delta_{n}(t) t p_{n} \frac{1-\left[1-\gamma+\gamma F\left(p_{n}\right)\right]^{n}}{n \gamma}
$$

which is a continuous function of $t$. Since the marginal entrant with $t=0$ has zero profit, and the marginal entrant with $t=1$ has profit $\pi^{o}$, for any $k \in\left(0, \pi^{o}\right), E(\pi \mid 0)<k$ and $E(\pi \mid 1)>k$. Therefore, there exists some $t_{f} \in[0,1)$ such that $E\left(\pi \mid t_{f}\right)=k$. That is, given $k$, there exists some $t_{f}$ such that potential entrants with $\beta_{i} \geq t_{f}$ will enter. Finally, from Proposition 5, the pricing strategy and consumer search behavior are optimal when there are $n$ sellers.

Different from the main model, here we have not proven that $t_{f}$ is an increasing function of $k$. The difficulty is that, as $t$ increases, equilibrium price decreases and thus the impact 
on the expected profit of the marginal seller with quality $t$ is unclear. ${ }^{12}$ For the rest of this section, we assume that (i) $N=2$ and (ii) $F$ and $G$ are both uniform distributions on $[0,1]$.

Then, it can be verified numerically that $t_{f}$ increases in $k$ for various values of $s$. Furthermore, consumer welfare initially increases but eventually decreases in $t$. The intuition is similar as in the main model: a lower $t_{f}$ leads to a higher expected number of sellers in the market but to a lower sellers' average quality. The increase in variety benefits consumers by expanding their search opportunities, whereas the decrease in quality harms consumers by reducing their search efficiency. However, here price is also affected, in two opposing directions: greater variety acts to reduce equilibrium prices, whereas lower quality works in the opposite direction as consumers search less due to the lower search benefit. Nevertheless, as in the main model, when $t_{f}$ is high, and thus the number of active sellers is low, the variety effect tends to dominate, so that a further increase in $t_{f}$ results in lower consumer welfare. On the other hand, when $t_{f}$ is low, the quality effect tends to dominate, so that an increase in $t_{f}$ results in higher consumer welfare.

Since $t_{f}$ increases in $k$, it follows that consumer welfare also first increases and then decreases in $k$. Given $s$, let $k^{*}$ be the entry cost that maximizes consumer welfare. Then, when $k \leq k^{*}$, same as in the main model, entry is excessive for both consumer and total welfare, whereas when $k>k^{*}$, entry is deficient for consumer welfare, and possibly also for total welfare.

Therefore, under additional functional and parameter restrictions, the results of our main model continue to hold in this alternative setting.

\section{CONCLUDING REMARKS}

In parallel to how free entry may lead to social inefficiency when firms possess market power, this paper has shown that unfettered entry can be detrimental to consumers when they have imperfect information about sellers' quality. In a model of consumer search with vertical and horizontal product differentiation, we find that there exists an inverted-U

\footnotetext{
${ }^{12}$ Recall that in the main model, equilibrium price is independent of $\gamma$.
} 
relationship between consumer welfare and entry cost (or the expected number of entrants). Free entry is excessive for both consumer and total welfare when entry cost is relatively low. It is also noteworthy that, in search markets, a decrease in average product quality can raise market price.

Our analysis is reminiscent of Akerlof (1970)'s classic model of the used-car market, where, under adverse selection, low-quality sellers drive out high-quality sellers, and the market may shut down completely. One may view our paper as taking Akerlof's model a step further by adding consumer search to it, so that a buyer can incur a search cost to find out, possibly with the help of an expert such as an auto mechanic, whether a car has a defect. ${ }^{13}$ A high-quality seller, whose car is less likely to be defective, then has a higher probability to succeed in trading, and hence may have more incentive to incur the (entry) cost to list its car for sale. The buyers' ability to detect a car's flaw through costly search may thus mitigate the adverse selection problem. But if entry cost is very low, it will not prevent low-quality sellers from entering the market; search efficiency for the buyers will then be too low and the market is likely to perform poorly. On the other hand, if entry cost is too high, very few sellers will enter the market, and even if their expected quality is high, it will be hard for buyers with heterogeneous preferences to find a match under the very limited search opportunities. This, in essence, is the trade-off between the variety and quality effects of entry in search markets, as uncovered in our paper. It should thus be clear that, while our model is highly stylized, its basic insights can be valid in more general settings and with alternative formulations of the market environment.

To illustrate our idea most transparently, we have abstracted from various market institutions that respond to the information problem and potentially improve the variety vs. quality trade-off. For example, firms may engage in costly advertising to convey quality information to consumers. While advertising cost has often been viewed as a barrier to competition, it may actually boost consumer welfare by deterring the entry of low-quality firms. Also, market intermediaries can simultaneously lower the number of entrants and

\footnotetext{
${ }^{13}$ This, together with the consumer's idiosyncratic taste, may then determine whether the car will meet her need.
} 
raise their average quality. Various accreditation agencies can serve this purpose, as, for instance, the accreditation of business schools could potentially help applicants search for the right MBA programs. An Internet platform may prominently display sellers who are more likely to meet consumers' needs, based on either organic search results or paid placement, as is done by the three largest search engines (Google, Yahoo! and Microsoft Bing). This can enhance consumer search efficiency, but also raises entry hurdles for less relevant sellers. Moreover, the organization of firms may also be motivated by such considerations. For instance, a hotel chain under a brand name may impose certain quality standards on its member hotels, and a merger between two firms might enable the merged firm to offer products that better meet consumer needs, both of which could help consumer search. To the extent that antirust and regulation can influence these business practices, it would be important for policy makers to recognize their beneficial roles.

Policies may also impact welfare directly by either facilitating or impeding entry. However, since it is unlikely that a policy maker will know the precise entry cost or entry scale that would be optimal for consumers, it is not obvious that government intervention would improve market outcomes, especially given the institutional arrangements that the market itself can make, as discussed above. Nevertheless, policies such as a minimum safety standard or truth-in-advertising regulation will likely improve search efficiency and benefit consumers.

\section{APPENDIX}

The Appendix contains proofs for Lemma 1, Lemma 2, and Proposition 5.

Proof of Lemma 1. (i) From (2), for all $t \in[0,1)$,

$$
\frac{d \gamma}{d t}=\frac{-t g(t)[1-G(t)]+g(t) \int_{t}^{1} x g(x) d x}{[1-G(t)]^{2}}=\frac{g(t)}{1-G(t)}(\gamma-t)>0 .
$$

(ii) Since

$$
\frac{d(t / \gamma)}{d t}=\frac{1}{\gamma^{2}}\left(\gamma-t \frac{d \gamma}{d t}\right)=\frac{1}{\gamma^{2}}\left[\gamma-\frac{g(t) t(\gamma-t)}{1-G(t)}\right]=\frac{\mu(t)}{\gamma^{2}}
$$

where $\mu(t) \equiv \gamma-\frac{g(t) t(\gamma-t)}{1-G(t)}$, to prove $\frac{d(t / \gamma(t))}{d t}>0$, it suffices to show $\mu(t)>0$ for all $t \in[0,1)$. 
Notice that $\mu(0)=\gamma>0$. Also, since

$$
\lim _{t \rightarrow 1} \frac{d \gamma}{d t}=\lim _{t \rightarrow 1} \frac{g(t)(\gamma-t)}{1-G(t)}=g(1) \lim _{t \rightarrow 1} \frac{(\gamma-t)}{1-G(t)}=g(1) \frac{\lim _{t \rightarrow 1} \frac{d \gamma}{d t}-1}{-\lim _{t \rightarrow 1} g(t)}=1-\lim _{t \rightarrow 1} \frac{d \gamma}{d t},
$$

we have $\lim _{t \rightarrow 1} \frac{d \gamma}{d t}=\frac{1}{2}$. It follows that

$$
\lim _{t \rightarrow 1} \mu(t)=1-g(1) \frac{\lim _{t \rightarrow 1} \frac{d \gamma}{d t}-1}{-g(1)}=\frac{1}{2} .
$$

Now, suppose to the contrary that $\mu(t) \leq 0$ for some $t \in(0,1)$. Then there must exist at least one $\hat{t} \in(0,1)$ such that $\mu(\hat{t})=0$ and $\mu^{\prime}(\hat{t})>0$. Our proof will be complete if we can show that this leads to a contradiction.

$$
\begin{aligned}
& \text { Rewrite } \mu(t)=(\gamma-t)\left[\frac{\gamma}{\gamma-t}-t \frac{g(t)}{1-G(t)}\right] \text {, then } \\
& \qquad \mu^{\prime}(t)=\left(\frac{d \gamma}{d t}-1\right)\left[\frac{\gamma}{\gamma-t}-t \frac{g(t)}{1-G(t)}\right]+(\gamma-t)\left[\frac{\frac{1}{\gamma^{2}} \mu(t)}{\left(1-\frac{t}{\gamma}\right)^{2}}-\frac{d\left(\frac{t g(t)}{1-G(t)}\right)}{d t}\right] .
\end{aligned}
$$

But for any $\hat{t} \in(0,1)$ such that $\mu(\hat{t})=0,\left[\frac{\gamma}{\gamma-\hat{t}}-\hat{t} \frac{g(\hat{t})}{1-G(\hat{t})}\right]=0$, and thus

$$
\mu^{\prime}(\hat{t})=-\left.(\gamma(\hat{t})-\hat{t}) \frac{d\left(\frac{t g(t)}{1-G(t)}\right)}{d t}\right|_{t=\hat{t}} \leq 0
$$

because $d\left(\frac{g(t)}{1-G(t)}\right) / d t \geq 0$ by assumption (1). This is a contradiction.

Proof of Lemma 2. First, we show that $\sum_{n=1}^{l} \delta_{n}(t)$ increases in $t$ for $l=1,2, \ldots, N$. Integrating by parts, we have

$$
\begin{aligned}
& \frac{(N-1) !}{(N-1-l) !(l-1) !} \int_{0}^{G(t)}\left(\xi^{N-1-l}\right)(1-\xi)^{l-1} d \xi \\
= & \frac{(N-1) !}{(N-l) !(l-1) !} \int_{0}^{G}(1-\xi)^{l-1} d\left(\xi^{N-l}\right) \\
= & \frac{(N-1) !}{(N-l) !(l-1) !}(1-G)^{l-1} G^{N-l}+\frac{(N-1) !}{(N-l) !(l-2) !} \int_{0}^{G} \xi^{N-l}(1-\xi)^{l-2} d \xi .
\end{aligned}
$$

Repeatedly performing integration by parts for $\int_{0}^{G} \xi^{N-l}(1-\xi)^{l-2} d \xi, \int_{0}^{G} \xi^{N-l+1}(1-\xi)^{l-3} d \xi$, 
and so on, we obtain:

$$
\begin{aligned}
& \frac{(N-1) !}{(N-1-l) !(l-1) !} \int_{0}^{G(t)}\left(\xi^{N-1-l}\right)(1-\xi)^{l-1} d \xi \\
= & \sum_{n=1}^{l} \frac{(N-1) !}{(n-1) !(N-n) !}(1-G(t))^{n-1} G(t)^{N-n}=\sum_{n=1}^{l} \delta_{n}(t) .
\end{aligned}
$$

Since $\int_{0}^{G(t)}\left(\xi^{N-1-l}\right)(1-\xi)^{l-1} d \xi$ increases in $G(t)$, which in turn increases in $t, \sum_{n=1}^{l} \delta_{n}(t)$ increases in $t$.

Then, for any $t^{\prime}>t$, recalling $\pi_{n}\left(t^{\prime}\right)>\pi_{n}(t)$ and $\pi_{n}(t)$ decreases in $n$, we have

$$
\begin{aligned}
E\left(\pi \mid t^{\prime}\right)-E(\pi \mid t) & =\sum_{n=1}^{N} \delta_{n}\left(t^{\prime}\right) \pi_{n}\left(t^{\prime}\right)-\sum_{n=1}^{N} \delta_{n}(t) \pi_{n}(t)>\sum_{n=1}^{N}\left[\delta_{n}\left(t^{\prime}\right)-\delta_{n}(t)\right] \pi_{n}(t) \\
& \geq \sum_{n=1}^{N}\left[\delta_{n}\left(t^{\prime}\right)-\delta_{n}(t)\right] \pi_{N}(t)=\left[\sum_{n=1}^{N} \delta_{n}\left(t^{\prime}\right)-\sum_{n=1}^{N} \delta_{n}(t)\right] \pi_{N}(t)>0 .
\end{aligned}
$$

Hence, $E(\pi \mid t)$ increases in $t$.

Proof of Proposition 5. (i) From (32), since $\frac{\partial \alpha}{\partial \gamma}>0$ and $\pi_{n i}^{\prime \prime}<0$,

$$
\frac{d p_{n}}{d \gamma}=-\frac{\frac{\partial \pi_{n i}^{\prime}}{\partial \gamma}+\frac{\partial \pi_{n i}^{\prime}}{\partial a} \frac{\partial \alpha}{\partial \gamma}}{\pi_{n i}^{\prime \prime}}<0 \text { if } \frac{\partial \pi_{n i}^{\prime}}{\partial \gamma}<0 \text { and } \frac{\partial \pi_{n i}^{\prime}}{\partial a}<0 .
$$

First, $\pi_{n i}^{\prime}$ in (32) can be rewritten as

$$
\begin{aligned}
\pi_{n i}^{\prime} & =[1-F(a)] \varphi_{n}+\int_{p_{n}}^{a}[1-\gamma+\gamma F(u)]^{n-1} d F(u)-p_{n}\left\{f(a) \varphi_{n}-\int_{p_{n}}^{a}[1-\gamma+\gamma F(u)]^{n-1} d f(u)\right\} \\
& =\left[1-F(a)-p_{n} f(a)\right] \varphi_{n}+\int_{p_{n}}^{a}[1-\gamma+\gamma F(u)]^{n-1}\left[f(u)+p_{n} f^{\prime}(u)\right] d u
\end{aligned}
$$

From (29), $\varphi_{n}=\frac{1}{n} \sum_{j=0}^{n-1}[1-\gamma+\gamma F(a)]^{j}$. Hence, $\frac{\partial \varphi_{n}}{\partial \gamma}<0$ and thus $\frac{\partial \pi_{n i}^{\prime}}{\partial \gamma}<0$.

Second, substituting $\varphi_{n}$ from (29), we have

$$
\begin{aligned}
& {[1-F(a)] \varphi_{n}+\int_{p_{n}}^{a}[1-\gamma+\gamma F(u)]^{n-1} f(u) d u } \\
= & {[1-F(a)] \frac{1-[1-\gamma+\gamma F(a)]^{n}}{n \gamma[1-F(a)]}+\frac{1}{n \gamma}\left\{[1-\gamma+\gamma F(a)]^{n}-\left[1-\gamma+\gamma F\left(p_{n}\right)\right]^{n}\right\} } \\
= & \frac{1-\left[1-\gamma+\gamma F\left(p_{n}\right)\right]^{n}}{n \gamma} .
\end{aligned}
$$


Thus, Letting $x \equiv 1-\gamma+\gamma F(a)$, we have

$$
\begin{aligned}
\gamma \pi_{n i}^{\prime}= & \frac{1-\left[1-\gamma\left(1-F\left(p_{n}\right)\right)\right]^{n}}{n}-p_{n}\left\{\frac{f(a)\left(1-x^{n}\right)}{n[1-F(a)]}-\gamma \int_{p_{n}}^{a}[1-\gamma(1-F(u))]^{n-1} d f(u)\right\} \\
\frac{\gamma}{p_{n}} \frac{\partial \pi_{n i}^{\prime}}{\partial a}= & {[1-\gamma+\gamma F(a)]^{n-1} \gamma f(a) \frac{f(a)}{1-F(a)}-\frac{1}{n}\left[1-[1-\gamma+\gamma F(a)]^{n}\right] \frac{d\left(\frac{f(a)}{1-F(a)}\right)}{d a} } \\
& \quad+\gamma[1-\gamma+\gamma F(a)]^{n-1} f^{\prime}(a)
\end{aligned}
$$

since $\frac{1-x^{n}}{1-x}=\sum_{j=0}^{n-1} x^{j}>n x^{n-1}$ for $x \in(0,1)$. Hence, $\frac{\partial \pi_{n i}^{\prime}}{\partial a}<0$.

(ii) Since $\pi_{n i}^{\prime \prime}<0$ and

$$
\frac{d p_{n}}{d n}=-\frac{\frac{\partial \pi_{n i}^{\prime}}{\partial n}}{\pi_{n i}^{\prime \prime}}
$$

$p_{n}$ decreases in $n$ if $\pi_{n i}^{\prime}$ decreases in $n$. From (32),

$$
\pi_{n i}^{\prime}=\left[1-F(a)-p_{n} f(a)\right] \varphi_{n}+\int_{p_{n}}^{a}[1-\gamma+\gamma F(u)]^{n-1}\left[f(u)+f^{\prime}(u) p_{n}\right] d u
$$

Hence, $\pi_{n i}^{\prime}$ decreases in $n$ if $\varphi_{n}$ decreases in $n$, which is indeed the case because, from (29) and recalling $x=1-\gamma+\gamma F(a) \in(0,1)$,

$$
\varphi_{n}-\varphi_{n+1}=\frac{1}{n} \sum_{j=0}^{n-1} x^{j}-\frac{1}{n+1} \sum_{j=0}^{n} x^{j}=\frac{1}{n+1}\left(\frac{1}{n} \sum_{j=0}^{n-1} x^{j}-x^{n}\right)>0 .
$$




\section{REFERENCES}

[1] Akerlof, G. (1970). "The Market for 'Lemons': Quality Uncertainty and the Market Mechanism." Quarterly Journal of Economics, Vol. 84, pp. 488-500.

[2] Amir, R. and Lambson, V. (2000). "On The Effects of Entry in Cournot Markets." Review of Economic Studies, Vol. 67(2), pp.235-254.

[3] Anderson, S. and Renault, R. (1999). "Pricing, Product Diversity, and Search Costs: A Bertrand-Chamberlin-Diamond Model." RAND Journal of Economics, Vol. 30 , pp. $719-735$

[4] Armstrong, M., Vickers, J., and Zhou, J. (2009). "Prominence and consumer search." RAND Journal of Economics, Vol. 40, No. 2, pp. 209-233.

[5] Athey, S. and Ellison, G. (2011). "Position Auctions with Consumer Search." Quarterly Journal of Economics, Vol. 126, pp. 1213-1270.

[6] Bar-Isaac, H, Caruana, G., and Cuñat, V. (2012). "Search, Design, and Market Structure." American Economic Review, Vol. 102(2), pp. 1140-1160.

[7] Cabral, L. (2004). "Simultaneous Entry and Welfare." European Economic Review, Vol. 48, pp. 943-957.

[8] Chen, Y. and He, C. (2012). "Paid Placement: Advertising and Search on the Internet." Economic Journal, Vol.121, pp. F309-28.

[9] Chen, Y. and Riordan, M. (2008). "Price-Increasing Competition." RAND Journal of Economics, Vol. 39, pp. 1042-1058.

[10] Diamond, P. (1971). "A Model of Price Adjustment." Journal of Economic Theory, Vol. 3, pp. $156-168$

[11] Eliaz, K. and Spiegler, R. (2012). "A Simple Model of Search Engine Pricing." Economic Journal, Vol.121, pp F329-39. 
[12] Haan, M. A. and Moraga-González, J. L. (2011). "Advertising for Attention in a Consumer Search Model." Economic Journal, Vol. 121: 552-579.

[13] Janssen, M. and Moraga-González, J. L. (2004). "Strategic Pricing, Consumer Search and the Number of Firms." Review of Economics Studies, Vol. 71, pp. 1089-1118.

[14] Mankiw, G. and Whinston, M. (1986). "Free Entry and Social Inefficiency." RAND Journal of Economics, Vol. 17, pp. 48-58.

[15] Satterthwaite, M.A. (1979). "Consumer Information, Equilibrium Industry Price, and the Number of Sellers." Bell Journal of Economics, Vol. 10, pp. 483-502.

[16] Seade, J. (1980). "On the Effects of Entry." Econometrica, Vol. 48, pp. 479-489.

[17] Stahl, D. (1989). "Oligopolistic Pricing with Sequential Consumer Search." American Economic Review, Vol. 79, pp. 700-712.

[18] Stigler, G. (1961). "The Economics of Information." Journal of Political Economy, Vol. 69, pp. $213-225$.

[19] Weitzman, M. L. (1979). "Optimal Search for the Best Alternative." Econometrica, Vol 47, pp. 641-654.

[20] Wolinsky, A. (1986). "True monopolistic competition as a result of imperfect information." Quarterly Journal of Economics, Vol. 101(3), pp. 493-511. 